\title{
Canine scent detection of canine cancer: a feasibility study
}

This article was published in the following Dove Press journal:

Veterinary Medicine: Research and Reports

26 October 2017

Number of times this article has been viewed

\author{
David C Dorman' \\ Melanie L Foster ${ }^{2}$ \\ Katherine E Fernhoff' \\ Paul R Hess ${ }^{2}$ \\ 'Department of Molecular and \\ Biomedical Sciences, ${ }^{2}$ Department \\ of Clinical Sciences, College of \\ Veterinary Medicine, North Carolina \\ State University, Raleigh, NC, USA
}

\begin{abstract}
The scent detection prowess of dogs has prompted interest in their ability to detect cancer. The purpose of this study was to determine whether dogs could use olfactory cues to discriminate urine samples collected from dogs that did or did not have urinary tract transitional cell carcinoma (TCC), at a rate greater than chance. Dogs with previous scent training $(n=4)$ were initially trained to distinguish between a single control and a single TCC-positive urine sample. All dogs acquired this task (mean $=15 \pm 7.9$ sessions; 20 trials/session). The next training phase used four additional control urine samples $(n=5)$ while maintaining the one original TCC-positive urine sample. All dogs quickly acquired this task (mean $=5.3 \pm 1.5$ sessions). The last training phase used multiple control $(n=4)$ and TCC-positive $(n=6)$ urine samples to promote categorical training by the dogs. Only one dog was able to correctly distinguish multiple combinations of TCC-positive and control urine samples suggesting that it mastered categorical learning. The final study phase evaluated whether this dog would generalize this behavior to novel urine samples. However, during double-blind tests using two novel TCC-positive and six novel TCC-negative urine samples, this dog did not indicate canine TCC-positive cancer samples more frequently than expected by chance. Our study illustrates the need to consider canine olfactory memory and the use of double-blind methods to avoid erroneous conclusions regarding the ability of dogs to alert on specimens from canine cancer patients. Our results also suggest that sample storage, confounding odors, and other factors need to be considered in the design of future studies that evaluate the detection of canine cancers by scent detection dogs. Keywords: urinary tract cancer, cancer detection dogs, cancer odor, olfactory memory, multiple sample learning
\end{abstract}

\section{Plain language summary}

Some studies suggest that dogs can detect cancer in human patients using odor cues. We evaluated the feasibility of using dogs to detect cancer in other dogs. We trained four scent detection dogs to distinguish urine samples from dogs with urinary tract cancer from urine collected from presumed normal dogs (controls). All scent detection dogs learned how to distinguish a few control and cancer urine samples. We then added more control and cancer urine samples to their training. We found that only one dog (Bud) mastered this larger training set and was able to reliably distinguish normal urine from urine samples collected from dogs with urinary tract cancer. Our final step was to evaluate whether "Bud" could reliably identify dogs with cancer when given new untrained cancer and control urine samples. We found that "Bud" could not reliably detect cancer in these new urine samples. Our results are similar to those of other scientists who trained dogs to detect prostate cancers in the urine of men. Future studies are needed to improve training methods to confirm whether dogs could be used to detect cancer in other dogs.
Correspondence: David C Dorman Department of Molecular Biomedical Sciences, College of Veterinary Medicine, North Carolina State University, 1060 William Moore Drive, Raleigh, NC 27607, USA

Tel +19195136237

$\mathrm{Fax}+19195136465$

Email david_dorman@ncsu.edu
Veterinary Medicine: Research and Reports 2017:8 69-76 (c) (7) (5) 2017 Dorman et al. This work is published and licensed by Dove Medical Press Limited. The full terms of this license are available at https://www.dovepress.com/terms. Php and incorporate the Creative Commons Attribution - Non Commercial (unported, v3.0) License (http://(creativecommons.org/licenses/by-nc/3.0/). By accessing the work you hereby accept the Terms. Non-commercial uses of the work are permitted without any further permission from Dove Medical Press Limited, provided the work is properly attributed. For permission for commercial use of this work, please see paragraphs 4.2 and 5 of our Terms (https://www.dovepress.com/terms.php). 


\section{Introduction}

Early detection of cancer reduces the need for aggressive treatment, decreases cancer metastasis, and improves survival in both human and veterinary patients. ${ }^{1,2}$ Approaches to detecting cancerous lesions frequently include clinical, radiological, or endoscopic examination of patients while a definitive cancer diagnosis often rests on histological examination of suspected abnormal tissue. ${ }^{3}$ Additional diagnostic approaches including the use of biomarkers have recently emerged for veterinary patients. ${ }^{4}$ Volatile organic compounds (VOCs) including alkane and aromatic compounds in exhaled breath, ${ }^{5}$ colon contents, ${ }^{6}$ and urine ${ }^{7}$ have been identified in human cancer patients using gas chromatography/mass spectroscopy. "Electronic noses" and other sensors that can detect VOCs and other chemicals found in biological samples from cancer patients have recently been developed. ${ }^{8-10}$

The conceptual basis behind an electronic nose originates in our knowledge of how olfactory neurons detect VOCs and other odorants. ${ }^{11}$ The intact olfactory system of animals functions like a chemical sensor: it detects, identifies, and discriminates different types of odorants. This function is well conserved across species leading to novel applications to cancer detection. For example, nematodes (Caenorhabditis elegans) display attractive chemotaxis toward human tumor cell lines, urine, and other samples collected from human cancer patients. ${ }^{12}$ Scent detection dogs have been trained to discriminate human cancer patient urine, exhaled breath, and other samples from those collected from control patient populations. ${ }^{13-22}$ These studies motivated our interest in evaluating the feasibility of using scent detection dogs to detect cancer in other dogs. Our study evaluated whether dogs could discriminate between urine samples collected from dogs with or without urinary tract transitional cell carcinoma (TCC; otherwise known as urothelial cell carcinoma [UCC]). We focused on TCC, since it is a highly invasive and relatively common form of cancer, accounting for approximately $2 \%$ of all reported canine cancers..$^{23}$ At diagnosis, canine TCCs are often advanced ${ }^{23}$ thus, methods that improve early detection may prove especially beneficial. The use of dogs for cancer detection is a relatively new noninvasive technology that, if effective, would be valuable for the screening for TCC and other cancers.

The purpose of this study was to determine whether scent detection dogs can be trained to alert specifically to urine samples from dogs with urinary tract TCC. We hypothesized that scent-trained dogs can discriminate between TCC and non-TCC canine patients at a rate greater than chance.

\section{Materials and methods \\ Scent detection dogs}

Research was performed in an AAALAC International accredited facility at North Carolina State University (NCSU). The NCSU Institutional Animal Care and Use Committee reviewed and approved all experimental protocols. The scent detection dogs used for this study were drawn from a stock of candidate scent detector dogs that were used in a previous study. We used four adult (25-28-month-old) male scent detection dogs (Marshall BioResources, North Rose, NY, USA) that had previously completed training on two olfactory discrimination (OD) tasks, namely vanillin versus an ethanol stimulus, and ammonium nitrate versus distractant odorants. The OD test uses a food rewarded conditioned stimulus (CS+) odorant and an unrewarded (CS-) odorant. ${ }^{24}$ Previous scent training with vanillin was initially used since it is a widely used odorant ${ }^{25}$ that provided a useful benchmark for the olfactory performance of the cohort of dogs used in the prior studies. Prior training with ammonium nitrate was part of an experimental study designed to assess the ability of dogs to detect improvised explosive devices (Gruen, unpublished observations, 2017). The use of dogs with prior scent training has been used by other investigations. ${ }^{24,26,27}$ Details on housing and husbandry procedures have been previously described. ${ }^{24}$

\section{OD testing}

Testing utilized the Toronto General Testing Apparatus (TGTA) which was originally developed for visual discrimination training with beagles (CanCog Technologies, Toronto, ON, Canada)..$^{28}$ The test system was modified to allow evaluation of larger breed dogs and has been previously described. ${ }^{29}$ Stainless steel bars separated the dog from the stimuli (Figure 1A). Openings in the bars allowed the dog to access the stimuli and obtain food rewards from the technician performing the test. The experimenter manipulated a black, sliding plastic stimulus presentation tray with three adjacent wells to hold and present the test stimuli to the dog (Figure 1B). Data were collected using DogCog ${ }^{\mathrm{TM}}$ software (CanCog Technologies) on a computer running a Windows 7 interface. The software recorded responses (as indicated by a keystroke from the experimenter), randomized stimulus, and reward positions and controlled trial timing.

Prior to training on this study, all dogs reacquired the vanillin-ethanol OD test. Dogs manipulated scented objects (petri dishes with $25 \mu \mathrm{L}$ urine) with their muzzle to receive a food reward (Pup-Peroni ${ }^{\circledR}$ Original bacon flavor treat; Del Monte Foods, San Francisco, CA, USA). Daily training 
sessions ( 20 trials/day) were performed 4-5 days/week until dogs reached criterion ( $\geq 80 \%$ correct on a single session or $\geq 70 \%$ correct on two consecutive sessions). Unless otherwise noted, this criterion was used for subsequent training phases.

Dogs were then trained to discriminate between urine samples collected from dogs with TCC (CS+) and urine samples collected from dogs with no evidence of TCC (CS-; Tables 1 and 2). Free catch urine samples collected from unfamiliar client owned dogs were either used fresh (within $6 \mathrm{~h}$ of collection) or divided $(250-500 \mu \mathrm{L})$ and stored at $-80^{\circ} \mathrm{C}$ until thawed and used. Each thawed aliquot was used on only one test day. The use of frozen samples was required since the rate at which individual dogs learned the OD task varied resulting in staggered entry of individual dogs into the different study phases. In addition, our access to control and TCC samples was intermittent; therefore, the majority $(>95 \%)$ of urine samples used on any individual test day were initially frozen and thawed prior to use. Subsequently, all urine samples were frozen and used in the study. Routine urinalyses, including microscopic evaluation of a wet preparation of the urine sediment, were performed on all samples. A diagnosis of urinary tract TCC was made by cytologic examination of either concentrated urine sediments or tumor samples acquired by traumatic catheterization. The following TCC training phases were used:

- Phase I: single control and single TCC-positive urine sample. Dogs were trained to discriminate between a urine sample from a clinically normal 5-year-old, castrated male (CM), golden retriever (CS-) and an 11-year-old, CM, Staffordshire bull terrier cross diagnosed with TCC (CS+).

- Phase II: multiple controls and a single TCC-positive urine sample. Dogs were trained to discriminate between four additional control urine samples from the original TCC urine sample.

- Phase III: multiple control ( $\mathrm{n}=4)$ and multiple TCC-positive ( $\mathrm{n}=6$ ) urine samples (categorical training). Categorical training involved using a larger training set consisting
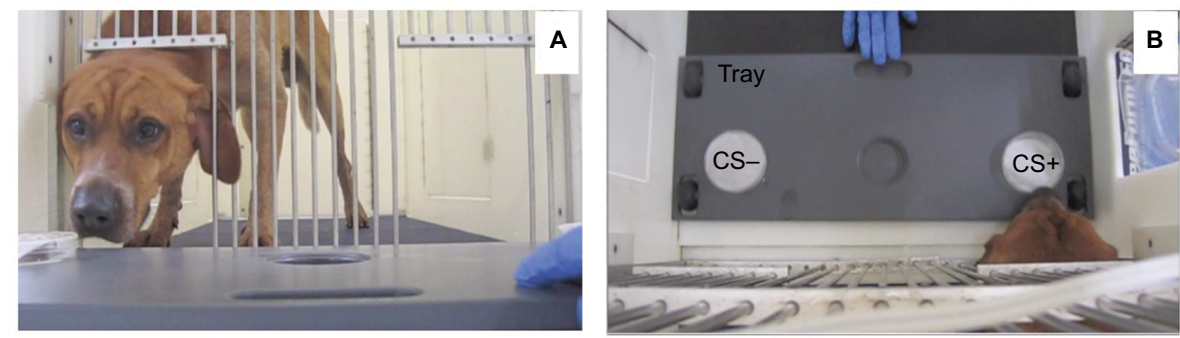

Figure I CanCog test apparatus.

Notes: Horizontal view (A) of a dog within the CanCog test apparatus as the tray with the control urine (CS-) and TCC-positive urine (CS+) samples were presented. A "birds eye" view of the test apparatus is also shown (B). Dogs are trained to move the petri dish with their muzzle to signify a response. Correct responses (CS+) are then rewarded by the individual performing the test.

Abbreviations: CS, conditioned stimulus; TCC, transitional cell carcinoma.

Table I Control urine samples used in the current experiment

\begin{tabular}{lllll}
\hline Phase & Age (years) & Sex & Breed & Underlying condition \\
\hline I, II, IV & 5 & MC & Golden retriever & None \\
II & 3 & MC & Labrador retriever mix & None \\
II & I.75 & MC & Pointer mix & Psychogenic PU/PD \\
II, III & 6 & MC & Doberman and hound cross & None \\
II, III & 2.5 & MC & Great Pyrenees & None \\
III & 4.5 & FS & Hound mix & Spay incontinence \\
III & 4.5 & FS & Hound mix & Lumbosacral pain \\
IV & II & FS & Greyhound & Vaginitis \\
IV & 3 & FS & German shepherd cross & None \\
IV & 7 & MC & Labrador retriever cross & None \\
IV & 6 & MC & Doberman and hound cross & Urinary tract infection \\
IV & I0 & FS & Labrador retriever & Urinary tract infection \\
IV & 7 & MC & Labrador retriever & \\
\hline
\end{tabular}

Notes: Training included: Phase I single control versus single TCC-positive, Phase II multiple control versus single TCC, and Phase III multiple control versus multiple TCCpositive samples. Phase IV involved presentation of novel urine samples during the final probe trials.

Abbreviations: FS, female spayed; MC, male castrated; PU/PD, polyuria/polydipsia; TCC, transitional cell carcinoma. 
Table 2 Urine samples from TCC-positive dogs used in the current experiment

\begin{tabular}{|c|c|c|c|c|}
\hline Phase & Age (years) & Sex & Breed & Other considerations \\
\hline I, II, III, IV' & II & MC & $\begin{array}{l}\text { Staffordshire bull } \\
\text { terrier mix }\end{array}$ & $\begin{array}{l}\text { Voided pre-chemotherapy sample. Concurrent Addison's disease and hypothyroidism. } \\
\text { Medications included Advantix, desoxycorticosterone pivalate, enalapril, levothyroxine, } \\
\text { and amlodipine. Diagnosis based on cytology from traumatic catheterization and the } \\
\text { presence of tumors during cystoscopic examination and ultrasonographic evidence of a } \\
\text { broad-based mass in the body of the urinary bladder }\end{array}$ \\
\hline III & II & FS & Rat terrier & $\begin{array}{l}\text { Voided sample. Medications included piroxicam, mitoxantrone ( } 28 \text { days earlier), } \\
\text { and famotidine. Diagnosis based on cytology from traumatic catheterization and } \\
\text { ultrasonographic evidence of a trigone and urethral mass }\end{array}$ \\
\hline III & II & FS & Beagle mix & $\begin{array}{l}\text { Voided pre-chemotherapy sample. Medications included glucosamine chondroitin and } \\
\text { Comfortis (spinosad). Diagnosis based on cytology and ultrasonographic evidence of a } \\
\text { trigone and urethral mass }\end{array}$ \\
\hline III & 14 & $M C$ & Keeshond & $\begin{array}{l}\text { Voided pre-chemotherapy sample. Diagnosis based on cytology and ultrasonographic } \\
\text { evidence of a trigone mass }\end{array}$ \\
\hline III & 12 & MC & Chihuahua & $\begin{array}{l}\text { Voided sample. Medications included piroxicam and mitoxantrone ( } 22 \text { days earlier). } \\
\text { Diagnosis based on cytology and ultrasonographic evidence of a trigone and bladder wall } \\
\text { mass }\end{array}$ \\
\hline III & 7 & FS & English pointer & $\begin{array}{l}\text { Voided pre-chemotherapy sample. Diagnosis based on cytology from traumatic } \\
\text { catheterization and ultrasonographic evidence of a trigone and urethral mass }\end{array}$ \\
\hline IV & 11 & FS & Beagle mix & $\begin{array}{l}\text { Voided sample. Medications included carboplatin ( } 19 \text { days earlier) and piroxicam. } \\
\text { Diagnosis based on cytology and ultrasonographic evidence of a urethral mass }\end{array}$ \\
\hline IV & 16 & FS & Chihuahua mix & $\begin{array}{l}\text { Voided pre-radiation sample. Concurrent hypothyroidism. Medications included } \\
\text { piroxicam, misoprostol, omeprazole, and clavamox. Diagnosis based on cytology from } \\
\text { traumatic catheterization and ultrasonographic evidence of urethral thickening with } \\
\text { extension into the trigone of the urinary bladder }\end{array}$ \\
\hline
\end{tabular}

Notes: Training included: Phase I single control versus single TCC-positive, Phase II multiple control versus single TCC-positive, and Phase III multiple control versus multiple TCC-positive samples. Phase IV involved presentation of novel urine samples during the final probe trials. ${ }^{2}$ Random inclusion of this urine sample allowed confirmation that the dogs were maintaining training throughout the study.

Abbreviations: FS, female spayed; MC, male castrated; TCC, transitional cell carcinoma.

of urine samples from four control dogs and six dogs with TCC (Tables 1 and 2). Samples were randomized within and across sessions. Daily training sessions (20 trials/ day) were performed 4-5 days/week until dogs reached criterion ( $\geq 80 \%$ correct on two consecutive sessions or $\geq 70 \%$ correct on three consecutive sessions).

The last phase assessed the ability of the one trained dog (Bud) to accurately respond to untrained TCC-negative $(n=6)$ and TCC-positive $(n=2)$ urine samples (probe trials). Unlike our previous phases, two of the six control urine samples came from dogs whose urinalysis indicated that there were bacteria (3 to $4+$ ), red blood cells ( 0 to $5-10)$, and white blood cells (0-5 to 5-10) present in the urine. Inclusion of these samples was intended to identify whether dogs may be responding to blood cells in the TCC-positive urine samples. This phase involved coded samples to blind the technician performing the task. Trials with coded samples $(n=10$ trials per session; 2-4 trials per sample) were unrewarded and interspersed with rewarded trials using previously used control (golden retriever) and TCC-positive (Staffordshire bull terrier cross) samples to maintain performance.

\section{Data analysis}

To measure individual dog performance, the percentage of correct decisions per session was calculated for each individual and each session. For example, in the initial learning tasks, a criterion of $80 \%$ correct ( $16 / 20$ correct responses in one session) corresponds to $P<0.01$ two-tailed binomial test versus chance. Descriptive statistics were calculated using a commercially available statistical program (JMP Pro 12.0.1; SAS Institute Inc., Cary, NC, USA). Unless otherwise indicated, data presented are mean \pm standard deviation.

\section{Results}

All dogs reacquired the vanillin-ethanol OD test (mean $=7.0 \pm 4.3$ sessions; range $3-15$ sessions; 20 trials $/$ session). Training success on the control urine versus TCC-positive urine OD task depended upon the phase of training and individual dog performance:

- Phase I: all dogs acquired this initial control urine versus TCC-positive urine OD task (mean $=15 \pm 7.9$ sessions; range 6-23 sessions; 20 trials/session). The rate of acquisition of this control versus TCC-positive urine was 
similar to that seen with the same cohort of four dogs when they were initially trained on the vanillin:ethanol OD test (mean $=12.3 \pm 4.3$ sessions; range $7-17$ sessions; Gruen, unpublished observation, 2017).

- Phase II: all dogs learned to distinguish multiple control urine samples from a previously learned TCC-positive sample, and the acquisition of this task was relatively rapid (mean $=5.3 \pm 1.5$ sessions; range $3-6$ sessions).

- Phase III (categorical learning): all dogs received 23 training sessions (460 trials) at which time only one dog (Bud) met criterion on this larger training set (Figure 2A) suggesting that it mastered categorical learning. Performance of the remaining three dogs varied considerably and often hovered near chance (53-59\%).

Figure 2B shows the results of the blinded trials using untrained TCC-positive urine samples with the one dog (Bud) that successfully completed all training phases. Despite its earlier success, this dog was unable to discriminate novel urine samples collected from TCC-positive dogs from TCC-negative dog urine (independent of the status of the urinalysis results). The overall success rate for "Bud" was $40 \%$ with success on the two TCC-positive samples of $0 \%$ to $75 \%$ (chance $=50 \%$ ).

\section{Discussion}

To our knowledge, this is the first study to assess whether scent detection dogs could be used to detect cancer in other dogs. As a feasibility study, we used a multi-stage training program that increased the number of control and TCCpositive urine samples. The final training phase used four control and six TCC-positive urine samples in order for the dogs to form a TCC odor "category." Categorization involves grouping different stimuli into categories or classes based on a shared stimulus property. ${ }^{30}$ This stimulus is not perceptually identical, but rather, possesses common features that lead to a common response to all related members of the class. When acquired, an individual responds to all stimuli in a class (e.g., TCC-positive urine samples) similarly based on the learning of a general "rule" that applies to all instances of the category, including novel instances. Category formation allows animals to respond appropriately to a novel, previously un-encountered stimuli without prior explicit training. We found that only one of four dogs trained on multiple control and TCC-positive urine samples successfully mastered this final training step suggesting that categorical learning may have occurred with this dog. Despite evidence suggesting that categorical learning occurred, this dog was unable to reliably distinguish novel control and TCC-positive urine samples during probe trials. Our results are qualitatively similar to a study that evaluated the ability of dogs to detect prostate cancer in people. ${ }^{15}$ That study used a much larger training set (50 prostate cancer samples and 67 control samples) during training. ${ }^{15}$ The prostate cancer study began with 10 dogs and eventually had only two dogs that could discriminate prostate cancer samples from controls during this initial training. Similar to our results, neither dog in this human study indicated prostate cancer samples more
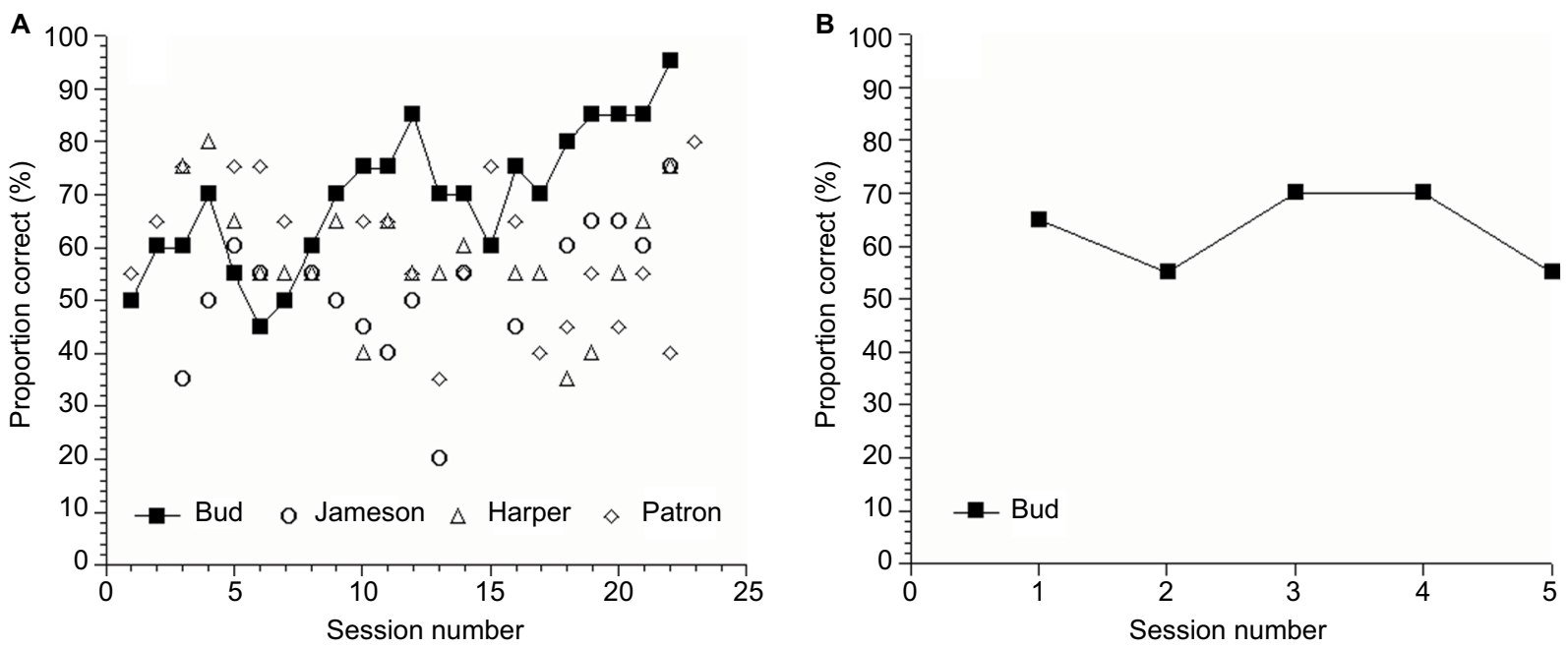

Figure 2 Performance of scent detection dogs.

Notes: Performance of dogs while being trained with urine samples from multiple TCC-positive and TCC-negative urine samples (A). Only one dog (Bud) reached criteria ( $\geq 80 \%$ correct during multiple sessions) during this phase. However, during double-blind tests using new urine samples, this dog did not indicate canine TCC-positive cancer samples more frequently than expected by chance (B). Each session consists of 20 trials.

Abbreviation: TCC, transitional cell carcinoma. 
frequently than expected by chance during double-blind tests that used novel samples. ${ }^{15}$ Collectively, these studies indicate an inherent challenge in the design of studies, namely how large a training set is required to develop the formation of a reliable cancer scent detection dog.

Another challenge investigators face with cancer scent detection studies is the ability of dogs to remember odors. ${ }^{31}$ One plausible explanation for the success of the one dog (Bud) to successfully complete the final training phase is that this dog learned to associate the individual urine odors with a food reward, thereby complicating the formation of a generalized common odor associated with cancer. Future studies evaluating canine cancer detection by scent detection dogs may need to evaluate larger training sets to confirm that a dog is responding to a TCC phenotype versus learning individual dog urine scents.

Our study also reinforces the importance of blinding technical staff when performing studies intended to confirm that a scent detector dog is signaling on a sample of interest. Although the impact of subtle cues between an animal and a handler, the so-called Clever Hans effect, has been known for well over a century, many studies involving scent detection in dogs fail to include this precaution. Lit et al ${ }^{32}$ dramatically showed how handler beliefs could affect the performance of scent detection dogs. These experimenters disclosed false locations of scent to handlers, which resulted in handlers reporting more alerts at these disclosed locations, despite no odorant being present in the test arena. ${ }^{32}$ Some studies evaluating cancer scent detection by dogs do not clearly indicate whether adequate blinding of research personnel has occurred, drawing into question the positive results of some published studies. ${ }^{33,34}$

Our study has some important limitations that could not be fully addressed in this feasibility study. For example, the scent detection dogs used in our study had similar genetic backgrounds and ages. It is also possible that the scent detection dogs could have mastered the TCC-positive urine:normal urine OD test with additional sessions or another training paradigm. A significant challenge we faced was obtaining a suitable number of urine samples from dogs with TCC at the time of their initial diagnosis. During the course of this 3-month study, we were only able to obtain 12 urine samples from two large referral veterinary medical hospitals. Future efforts may want to consider choosing other cancer types with a higher incidence rate. In any case, sample handling and storage will likely need to be considered in future canine cancer detection studies. For example, in our study, some aliquots of the urine samples collected from the Staffordshire bull terrier cross and golden retriever were frozen and subsequently used in the last phase of the study. We did not observe any apparent change in the response rate of the trained dogs to these thawed frozen samples. It remains unknown however whether freezing of urine samples may affect the odor signature of some urine samples. The "background" odor signature from dog urine could vary depending upon diet, sex, reproductive status, and the presence or absence of urinary drug (e.g., nonsteroidal anti-inflammatory drugs [NSAIDs], antibiotics, chemotherapeutics, and ectoparasite medications) metabolites, proteins, epithelial cells, blood, and other materials in urine. These potentially confounding factors were variably present in the TCC-positive dogs used in our current study (Table 2) and may have impaired effective categorical learning on the part of the scent detection dogs. Standardizing diets and treatments to control for these potential confounding factors in future studies will likely remain elusive for future investigators. Finally, at the time the urine samples were collected, we relied upon a presumptive diagnosis of TCC. This diagnosis was based upon cytologic evaluation of urine sediment and ultrasonographic evidence of the presence of thickening of the urethra and trigone region of the bladder or the presence of a mass in the bladder. Confirmation of a diagnosis of TCC often rests on histopathological examination of affected tissues. ${ }^{23}$ Biopsy results were not available for the individual dogs used in the study, and the dogs were lost to long-term follow-up and necropsy.

Despite these limitations, our study provides important new information concerning the feasibility of using scent detection dogs to detect cancer in other dogs. This study is especially timely given the increasing interest this topic has garnered among the general public, veterinarians, and other health professionals. Additional research including efforts to identify optimal sample handling, storage, and number of samples is needed, however, before this approach can be applied as a screening tool for veterinary oncology.

\section{Conclusion}

Using scent detection dogs for the identification of cancer has been the subject of numerous media reports. However, the scientific literature concerning this topic remains limited. Our study represents a novel attempt to use scent detection dogs to identify TCC in other dogs. The results of this study suggests that the ability of individual scent detection dogs to learn an olfactory discrimination test in which they have to distinguish between urine samples collected from clinically 
normal dogs and dogs with TCC varies considerably. Our results also suggest that large training sets, in excess of the one used in this pilot study, may be required to adequately train scent detection dogs for this purpose. These results suggest that the use of dogs for the scent detection of cancer in other dogs remains a significant clinical challenge in part because of variability in individual scent detection dog's ability to learn an appropriate olfactory task, urine sample storage, and other confounding factors that could impair canine performance.

\section{Acknowledgments}

The authors appreciate the assistance of the medical oncology service at NCSU and Dr. Laurel Williams of the Veterinary Specialty Hospital of the Carolinas for their assistance with sample collection. Dr. Katherine Fernhoff's current affiliation is VCA Northwest Veterinary Specialists, Clackamas, OR 97015, USA. This study was originally presented at the Consortium for Canine Comparative Oncology Symposium in Durham, NC, USA.

\section{Disclosure}

The authors report no conflicts of interest in this work.

\section{References}

1. Neal RD, Tharmanathan P, France B, et al. Is increased time to diagnosis and treatment in symptomatic cancer associated with poorer outcomes? Systematic review. Br J Cancer. 2015;112(suppl):S92-S107.

2. Schleis SE. Cancer screening tests for small animals. Vet Clin North Am Small Anim Pract. 2014;44:871-881.

3. Rhind SM. Veterinary oncological pathology - current and future perspectives. Vet J. 2002;163(1):7-18.

4. Henry CJ. Biomarkers in veterinary cancer screening: applications, limitations and expectations. Vet J. 2010;185(1):10-14.

5. Sun X, Shao K, Wang T. Detection of volatile organic compounds (VOCs) from exhaled breath as noninvasive methods for cancer diagnosis. Anal Bioanal Chem. 2016;408(11):2759-2780.

6. de Boer NK, de Meij TG, Oort FA, et al. The scent of colorectal cancer: detection by volatile organic compound analysis. Clin Gastroenterol Hepatol. 2014;12(7):1085-1089.

7. Amann A, Costello Bde L, Miekisch W, et al. The human volatilome: volatile organic compounds (VOCs) in exhaled breath, skin emanations, urine, feces and saliva. J Breath Res. 2014;8:034001.

8. Brooks SW, Moore DR, Marzouk EB, Glenn FR, Hallock RM. Canine olfaction and electronic nose detection of volatile organic compounds in the detection of cancer: a review. Cancer Invest. 2015;33(9):411-419.

9. Capelli L, Taverna G, Bellini A, et al. Application and uses of electronic noses for clinical diagnosis on urine samples: a review. Sensors (Basel). 2016;16(10):1708.

10. Westenbrink E, Arasaradnam RP, O'Connell N, et al. Development and application of a new electronic nose instrument for the detection of colorectal cancer. Biosens Bioelectron. 2015;67:733-738.

11. Wilson AD, Baietto M. Advances in electronic-nose technologies developed for biomedical applications. Sensors (Basel). 2011;11(1):1105-1176.
12. Hirotsu T, Sonoda H, Uozumi T, et al. A highly accurate inclusive cancer screening test using Caenorhabditis elegans scent detection. PLoS One. 2015;10(3):e0118699.

13. Cornu JN, Cancel-Tassin G, Ondet V, Girardet C, Cussenot O. Olfactory detection of prostate cancer by dogs sniffing urine: a step forward in early diagnosis. Eur Urol. 2011;59(2):197-201.

14. Ehmann R, Boedeker E, Friedrich U, et al. Canine scent detection in the diagnosis of lung cancer: revisiting a puzzling phenomenon. Eur Respir J. 2012;39(3):669-676.

15. Elliker KR, Sommerville BA, Broom DM, Neal DE, Armstrong S, Williams HC. Key considerations for the experimental training and evaluation of cancer odour detection dogs: lessons learnt from a double-blind, controlled trial of prostate cancer detection. BMC Urol. 2014;14:22.

16. Guerrero-Flores H, Apresa-García T, Garay-Villar Ó, et al. A noninvasive tool for detecting cervical cancer odor by trained scent dogs. BMC Cancer. 2017;17(1):79.

17. Hackner K, Errhalt P, Mueller MR, et al. Canine scent detection for the diagnosis of lung cancer in a screening-like situation. J Breath Res. 2016;10(4):046003.

18. McCulloch M, Jezierski T, Broffman M, Hubbard A, Turner K, Janecki T. Diagnostic accuracy of canine scent detection in early- and late-stage lung and breast cancers. Integr Cancer Ther. 2006;5(1):30-39.

19. Pickel D, Manucy GP, Walker DB, Hall SB, Walker JC. Evidence for canine olfactory detection of melanoma. Appl Anim Behav Sci. 2004;89(1-2):107-116.

20. Sonoda H, Kohnoe S, Yamazato T, et al. Colorectal cancer screening with odour material by canine scent detection. Gut. 2011;60(6):814-819.

21. Taverna G, Tidu L, Grizzi F, et al. Olfactory system of highly trained dogs detects prostate cancer in urine samples. J Urol. 2015;193(4):1382-1387.

22. Willis CM, Church SM, Guest CM, et al. Olfactory detection of human bladder cancer by dogs: proof of principle study. BMJ. 2004; 329(7468):712.

23. Fulkerson CM, Knapp DW. Management of transitional cell carcinoma of the urinary bladder in dogs: a review. Vet J. 2015;205(2):217-225.

24. Lazarowski L, Foster ML, Gruen ME, et al. Olfactory discrimination and generalization of ammonium nitrate and structurally related odorants in Labrador retrievers. Anim Cogn. 2015;18(6):1255-1265.

25. Frasnelli J, Hummel T, Berg J, Huang G, Doty RL. Intranasal localizability of odorants: influence of stimulus volume. Chem Senses. 2011;36(4): 405-410.

26. Hall NJ, Smith DW, Wynne CD. Effect of odor preexposure on acquisition of an odor discrimination in dogs. Learn Behav. 2014;42(2):144-152.

27. Marchal S, Bregeras O, Puaux D, Gervais R, Ferry B. Rigorous training of dogs leads to high accuracy in human scent matching-to-sample performance. PLoS One. 2016;11(2):e0146963.

28. Milgram NW, Head E, Weiner E, Thomas E. Cognitive functions and aging in the dog: acquisition of nonspatial visual tasks. Behav Neurosci. 1994;108(1):57-68.

29. Lazarowski L, Foster ML, Gruen ME, et al. Acquisition of a visual discrimination and reversal learning task by Labrador retrievers. Anim Cogn. 2014;17(3):787-792.

30. Lazareva OF, Wasserman EA. Effects of stimulus duration and choice delay on visual categorization in pigeons. Learn Motiv. 2009;40(2):132-146.

31. Williams M, Johnston JM. Training and maintaining the performance of dogs (Canis familiaris) on an increasing number of odor discriminations in a controlled setting. Appl Anim Behav Sci. 2002;78(1):55-65.

32. Lit L, Schweitzer JB, Oberbauer AM. Handler beliefs affect scent detection dog outcomes. Anim Cogn. 2011;14(3):387-394.

33. Edwards TL, Browne CM, Schoon A, et al. Animal olfactory detection of human diseases: guidelines and systematic review. J Vet Behav. 2017;20:59-73.

34. Pirrone F, Albertini M. Olfactory detection of cancer by trained sniffer dogs: a systematic review of the literature. JVet Behav. 2017;19:105-117. 


\section{Publish your work in this journal}

Veterinary Medicine: Research and Reports is an international, peer-reviewed, open access journal publishing original research, case reports, editorials, reviews and commentaries on all areas of veterinary medicine. The manuscript management system is completely online and includes a very quick and fair peer-review system.
Visit http://www.dovepress.com/testimonials.php to read real quotes from published authors.

Submit your manuscript here: http://www.dovepress.com/veterinary-medicine-research-and-reports-journal 